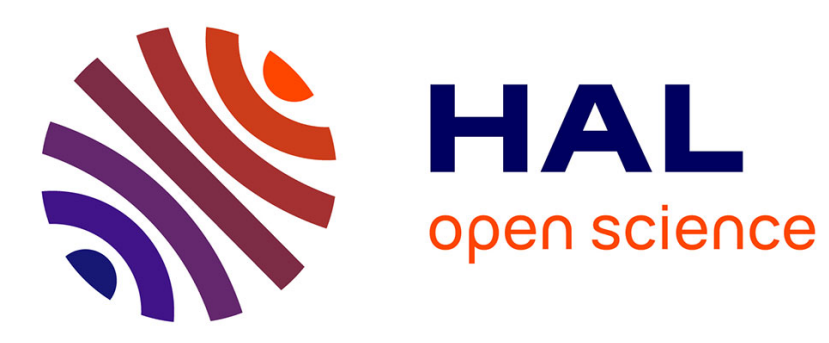

\title{
SmEdA Vibro-Acoustic Modeling of a Trimmed Truck Cab in the Mid-Frequency Range
}

Laurent Maxit, Jean-Louis Guyader, Kerem Ege, Youssef Gerges, Ha Dong Hwang, Céline Sandier

\section{To cite this version:}

Laurent Maxit, Jean-Louis Guyader, Kerem Ege, Youssef Gerges, Ha Dong Hwang, et al.. SmEdA Vibro-Acoustic Modeling of a Trimmed Truck Cab in the Mid-Frequency Range. 10th International Styrian Noise, Vibration \& Harshness Congress: The European Automotive Noise Conference, Jun 2018, Graz, Austria. pp.2018-01-1506, 10.4271/2018-01-1506 . hal-01829435

\section{HAL Id: hal-01829435 https://hal.science/hal-01829435}

Submitted on 18 Jul 2018

HAL is a multi-disciplinary open access archive for the deposit and dissemination of scientific research documents, whether they are published or not. The documents may come from teaching and research institutions in France or abroad, or from public or private research centers.
L'archive ouverte pluridisciplinaire $\mathbf{H A L}$, est destinée au dépôt et à la diffusion de documents scientifiques de niveau recherche, publiés ou non, émanant des établissements d'enseignement et de recherche français ou étrangers, des laboratoires publics ou privés. 


\section{SmEdA Vibro-Acoustic Modeling of a Trimmed Truck Cab in the Mid-Frequency Range}

Laurent Maxit, Jean-Louis Guyader, Kerem Ege, Youssef Gerges, Ha Dong Hwang and Céline Sandier, INSA Lyon / LVA

Citation: Maxit, L., Guyader, J.-L., Ege, K., Gerges, Y. et al., "SmEdA Vibro-Acoustic Modeling of a Trimmed Truck Cab in the Mid-Frequency Range," SAE Technical Paper 2018-01-1506, 2018, doi:10.4271/2018-01-1506 


\title{
SmEdA vibro-acoustic modeling of a trimmed truck cab in the mid-frequency range
}

\author{
Author, co-author (Do NOT enter this information. It will be pulled from participant tab in \\ MyTechZone) \\ Affiliation (Do NOT enter this information. It will be pulled from participant tab in MyTechZone)
}

\begin{abstract}
The City Lightweight and Innovative Cab (CLIC) project was a scientific collaboration gathering public and private organizations. The aim was to propose an innovative lighten truck cab, where a high strength steel was used. As long as it could affect directly the acoustic environment of the cab, it was necessary to be able to simulate the vibroacoustic behavior of the truck cab in the mid frequency range. The dissipative treatments used for noise and vibration control such as viscoelastic patches and acoustic absorbing materials must then be taken into account in the problem. A process based on the SmEdA (Statistical modal Energy distribution Analysis) method was developed and is presented in this paper. SmEdA allows us substructuring the global problem, to study the interaction between the floor and the interior cavity. The process consists in building finite element models (FEM) of each subsystem (floor, internal cavity), including the dissipative material (damping layer, poroelastic material). Standard modal FEM calculations are then performed for each uncoupled subsystem. From the spatial mode shapes, and the modal strain -kinetic energies, the modal loss factors of both subsystems are estimated. Finally, the pressure levels inside the cavity are deduced from the resolution of the SmEdA equations. To validate this process, a truck cabin has been excited mechanically on a rail of the floor and the pressure levels at different positions inside the cabin were measured for different configurations of dissipative treatment. Comparisons between SmEdA and experimental results allows us to assess the accuracy of the proposed method
\end{abstract}

\section{I-Introduction}

With the new security and pollution standards, the industrials have to make more effort on their engines design. The CLIC (City Lightweight and Innovative $\mathrm{Cab}$ ) project, that is gathering different private and public partners, aims to suggest a new design for lighten cabin truck, where a high strength steel is used. This lightening process decreases the $\mathrm{NVH}$ performances, and the mid frequency vibroacoustic behavior should be taken into account to ensure the driver comfort. The finite element method (FEM) is the most common modeling method, despite the drawbacks in time consuming and computer memory requirements. The SEA method (Statistical Energy Analysis) [1] is well used for the high frequency studies, but it requires the validation of many hypotheses [2]. The SmEdA method (Statistical modal Energy distribution Analysis) [35] may be seen as a good compromise between those two methods. It consists to decompose the global system in different subsystems.

Page 1 of 8
Then, each subsystem (taken separately) is described by its uncoupled (free or blocked) modes. FEM can be used to calculate the subsystem modes when the geometry of the considered subsystem is complex. Then, the modal coupling loss factors describing the coupling between the pair of modes are deduced. Considering the modal injected power by the external source and writing the energy equilibrium of each mode, the energy response of the global system can be deduced. Contrary to the SEA method, it does not assume the equipartition of the modal energies. The method is then well adapted for dealing vibro-acoustic problems in the midfrequency range. In particular, it can describe non-resonant transmission [4] and take into account the effect of spatially localized excitations when the modal overlap factor is low [3] or inhomogeneity of the vibratory field within a heterogeneous subsystem [5,6]. Even if SmEdA is not implemented in an industrial code, it is easily to build a model with a programming language like MATLAB associated with input data from a commercial FEM code like NASTRAN.

In this work, one focuses on the modelling of the vibroacoustic behavior of a truck cab in the mid-frequency range in order to assess its NVH performances. In this analysis, the effect of the vibration and noise control treatments (trim) should be considered. It may be the visco-elastic layers used for damped the structural vibration or the porous elastic materials used for their sound absorbing ability. An approach based on SmEdA for taking into account these dissipative treatments has been recently developed during the $\mathrm{PhD}$ thesis of $\mathrm{Ha}$ Dong Hwang [7]. Basically, this method consists to develop an equivalent model of the dissipative materiel in order to be easily implemented in the subsystem FE model, to extract the subsystem modes of the dissipative subsystem and then to estimate the modal damping loss factors using a projection of the complex finite element matrices. These modal loss factors characterize in SmEdA the effect of the dissipative material which depend on its positions, its geometry and its mechanical characteristics. This methodology will be reminded and illustrated on a basic case on section II. Then, one proposes its application for describing the interaction between the floor and the passenger cavity of truck cabin (i.e. the transmission through the lateral faces are supposed negligible). Results are presented in section III. Finally, after resolving the SmEdA equations, one can deduce the pressure level on the driver ears. The numerical results are compared to experimental tests to study its accuracy. 


\section{II-Statistical Modal Energy Distribution Analysis}

\section{II.1 Principle}

SmEdA [3-7] is based on a modal description of each subsystems and it predicts the power flow exchanged between these different subsystem modes. Let us considers two subsystems denoted $\# 1$ and \#2, composed of $N_{1}$ and $N_{2}$ modes respectively in the considered frequency band of excitation. The SmEdA equations expressing the energy conservation for each mode is written as:

$$
\left\{\begin{array}{r}
\Pi_{i n j}^{1 p}=\left(\omega_{p}^{1} \eta_{p}^{1}+\sum_{q^{\prime}=1}^{N_{2}} \beta_{p q^{\prime}}^{12}\right) E_{p}^{1}-\sum_{q^{\prime}=1}^{N_{2}} \beta_{p q^{\prime}}^{12} E_{q^{\prime}}^{2}, \\
\forall p \in\left[1, \ldots, N_{1}\right], \\
\Pi_{i n j}^{2 q}=-\sum_{p^{\prime}=1}^{N_{1}} \beta_{p^{\prime} q}^{12} E_{p^{\prime}}^{1}+\left(\omega_{q}^{2} \eta_{q}^{2}+\sum_{p^{\prime}=1}^{N_{1}} \beta_{p^{\prime} q}^{12}\right) E_{q}^{2}, \\
\forall q \in\left[1, \ldots, N_{2}\right],
\end{array}\right.
$$

where : $\quad-\Pi_{i n j}^{\alpha m}$ is the injected power into mode $m$ of subsystem $\alpha(\alpha=1,2)$;

$$
\text { - } E_{m}^{\alpha}, \omega_{m}^{\alpha} \text { and } \eta_{m}^{\alpha} \text { are respectively the energy, the }
$$

angular frequency and the damping loss factor of mode $m$ of subsystem $\alpha$, and;

$$
\text { - } \beta_{m n}^{12} \text { is the modal coupling loss factor between the }
$$

mode $m$ of subsystem 1 and the mode $n$ of the subsystem 2 :

$$
\begin{aligned}
& \beta_{m n}^{12}=\left(\mathrm{W}_{m n}^{12}\right)^{2} \times \ldots \\
& \frac{\omega_{m}^{1} \eta_{m}^{1}\left(\omega_{n}^{2}\right)^{2}+\omega_{n}^{2} \eta_{n}^{2}\left(\omega_{m}^{1}\right)^{2}}{\left[\left(\omega_{m}^{1}\right)^{2}-\left(\omega_{n}^{2}\right)^{2}\right]^{2}+\left(\omega_{m}^{1} \eta_{m}^{1}+\omega_{n}^{2} \eta_{n}^{2}\right)\left[\omega_{m}^{1} \eta_{m}^{1}\left(\omega_{n}^{2}\right)^{2}+\omega_{n}^{2} \eta_{n}^{2}\left(\omega_{m}^{1}\right)^{2}\right]},
\end{aligned}
$$

where $\mathrm{W}_{m n}^{12}$ is the intermodal work between the mode $m$ of subsystem 1 and the mode $n$ of the subsystem 2 . This latter term expresses the spatial matching between the two modes and it can be evaluated from the modeshapes on the coupling surface (see [3]).

In these equations, the angular frequencies $\omega_{m}^{\alpha}$ and the intermodal works $\mathrm{W}_{m n}^{12}$ can then be deduced from an extraction of the subsystem modes using a standard finite element code. Usually, for the modal damping loss factors, a constant value is considered for all the modes of a given subsystem. This approach is however not adapted to describe the effect of dissipative material. A methodology will be described in the next section to estimate each of these modal loss factors from the characteristics and the position of the dissipative material inside the subsystem.
For illustration, one considers a white noise point source on subsystem 1 in the following. $M$ is the point of excitation and $S_{F F}$ is the density power spectrum of the white noise signal of the excitation. The modal injected power is then given by:

$$
\Pi_{i n j}^{1 p}=\frac{S_{F F}\left[\phi_{p}^{1}(M)\right]^{2}}{4}, \Pi_{i n j}^{2 q}=0,
$$

where $\phi_{p}^{1}(M)$ is the $p^{\text {th }}$ mode shape at point $M$ of subsystem 1 .

Knowing the injected power and the modal characteristics of each subsystem, one can compute the modal energies by resolving Eq. (1), and then deduce the total energy of each subsystem,

$$
E_{1}=\sum_{p=1}^{N_{1}} E_{p}^{1}, \quad E_{2}=\sum_{q=1}^{N_{2}} E_{q}^{2}
$$

and the spatial mean square of the pressure for an acoustic subsystem or of the vibratory velocity for a mechanical subsystem:

$$
<p_{\alpha}{ }^{2}>=\frac{\rho_{0} c_{0}^{2} E_{\alpha}}{V_{\alpha}},\left\langle V_{\alpha}^{2}>=\frac{E_{\alpha}}{M_{\alpha}} .\right.
$$

\section{II.2 Process for taking the dissipative treatments into account}

In this section, we present the outline of the process allowing us to include dissipative material in the SmEdA modelling. The details can be found in Ref. [7].

The process can be decomposed in four steps:

- The first step consists in representing the dissipative materials by using homogenized equivalent material models.

For a mechanical subsystem, an equivalent single layer model may be used for representing the viscoelastic layer patches (as illustrated on Fig. 2). The method proposed in [8] based on the zigzag theory and the traveling wave approach gives us the equivalent complex Young modulus and the equivalent Poisson's ratio of the multilayer from the knowledge of the thickness and mechanical parameters of each layer. This approach permit to deal with any number of layers and facilitates the modification of the material properties of each layer. It also facilitates the positioning of the viscoelastic patches on the structure by modifying the element properties of the 2D shell finite element model of the structure.

For an acoustic subsystem, the well-known equivalent fluid model can be used to represent the acoustic behavior of the poroelastic materiel. The complex equivalent fluid density and the complex equivalent fluid compressibility can be estimated from acoustic tube measurements with the Utsuno two cavities method [7].

This type of modelling has two main advantages: (a) it permits increasing the upper frequency bound of the FEM calculation compared to a full detailed model; (b) it is well adapted for 
optimizing dissipative materials. Different positions of the dissipative materials could be considered easily by modifying only the property of the element in the original finite element mesh;

- The second step consists in building the finite element models of each subsystem treated. The dissipative treatments are introduced in these models by using the homogenized material models. As the mechanical properties of these materials may vary with frequency, constant mean values are considered for each frequency band of calculation (typically, third-octave band). A finite element model is then created for each subsystem and each frequency band. Boundary conditions at the coupling surface are defined in accordance with DMF [3]. The normal modes of each subsystem and each frequency band are then extracted. The natural frequencies and mode shapes at the coupling junction (normalized to a unit modal mass) are saved in the database used for the SmEdA calculation;

- The modal damping loss factors are estimated in the third step. Modal equations of each subsystem containing a dissipative material are obtained from the dynamic equation of motion of the subsystem considered and the Galerkin method with normal mode shapes is used. Cross-modal terms are then neglected for estimating the modal damping loss factors by analogy between the modal equations obtained and the standard ones. Analytical expressions of the modal damping loss factors are obtained in function of the subsystem modeshapes and the complex matrices of the finite element model of the dissipative subsystem. These theoretical developments are described in details in [7]. These calculations may be achieved in post-processing of the modal extraction calculation and they are few time-consuming;

- The final step consists in resolving the SmEdA equations (1) depending on the subsystem modal information estimated numerically in the two previous steps. Finally, the total energy of each subsystem can be estimated by summing the modal energies (4).

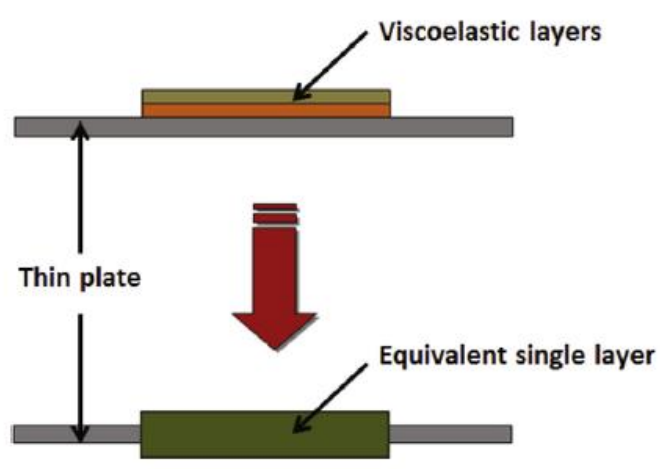

Figure 1. Schematic representation of equivalent single layer modelling for a plate treated with viscoelastic materials.

\section{II.3 Illustration on a laboratory test case}

For illustrating the process, let us considered the steel plate-cavity system excited by a mechanical force as presented in Fig. 2. Three configurations of damped are considered: the bare case (without any viscoelastic treatments); the viscol plate damped by one viscoelastic patch, and; the visco2 plate damped by two viscoelactic patches. The viscoelastic layer is a $2.6 \sim \mathrm{mm}$ thick prefabricated damping patch currently used in the automotive industry. It can be semi-permanently attached to the plate by heating.
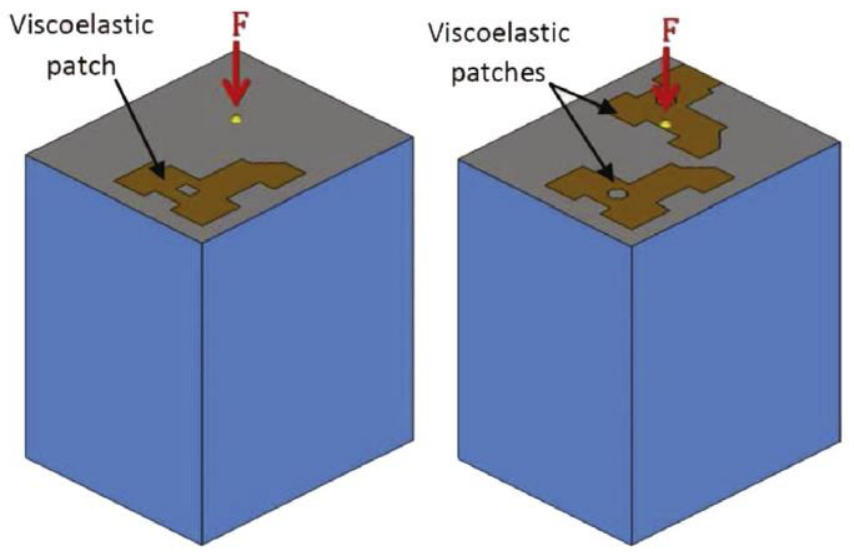

Figure 2. Plate-cavity system excited by a mechanical point force. Two damped configurations: (a), viscol case ; (b), visco2 case.

The mechanical properties of the equivalent single layer model of the viscoelastic layer patch are obtained using the inhouse code MOVISAND based on the zig-zag method [8]. The complex elastic modulus of the viscoelastic material is determined using the timetemperature superposition principle. The frequency-dependent curves of isothermal properties at different temperatures were estimated experimentally by the manufacturer using a dynamic mechanical analyser (DMA+300 from METRAVIB company). For information, the damping loss factors of the viscoelastic layer and the steel are plotted on Fig. 3 for each third octave band.

Once the parameters of the equivalent models for the viscoelastic multilayer, a finite element mesh of each subsystem was created. The plate was represented with 13776 quadrilateral shell elements whereas the cavity was represented with 4031412 tetrahedral solid elements. The mesh sizes were chosen in order to have a minimum of six elements per wavelength at $8 \mathrm{kHz}$. The modal analysis was performed for each subsystem for each third octave band using the MSC/NASTRAN code and the Lanczos method. A NASTRAN DMAP program allowed estimating the modal damping loss factors in the same run as the modal analysis.

Figure 3 shows the modal damping loss factors for the two damped plates. It can be seen that the damping pads provide substantially high damping compared to the bare plate. Their effects decrease slightly with increasing frequencies, similar to the decreasing values of the damping loss factors of the equivalent viscoelastic model. The damping values can vary substantially from one mode to another for the same configuration. This indicates that some modes exhibiting low strains at the pad positions are less influenced by the damping pad than others. This is illustrated in Fig. 4 where the spatial shapes of two modes of viscol plate (identified by two red circles on Fig. 3) are plotted: the first has a natural frequency of $1584 \mathrm{~Hz}$ and a damping loss factor of 0.007 , whereas the second has a natural frequency of $1818 \mathrm{~Hz}$ and a damping loss factor of 0.05 . It can be clearly seen that the first mode does not exhibit significant spatial deformation around the damping pad, contrary to the second mode. Compared to the viscol plate, the visco 2 plate presents averaged

Page 3 of 8 
values roughly $50 \%$ higher and lower variations from one mode to another. This can be explained by the fact that the two damping pads occupied an area twice as large as a single pad.

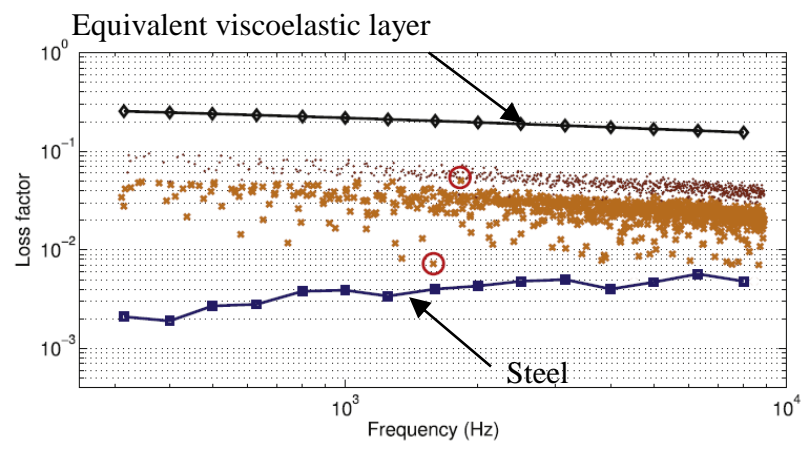

Figure 3. Plate modal damping loss factors as a function of the modal frequency: cross, visco 1 plate; point, visco2 plate.

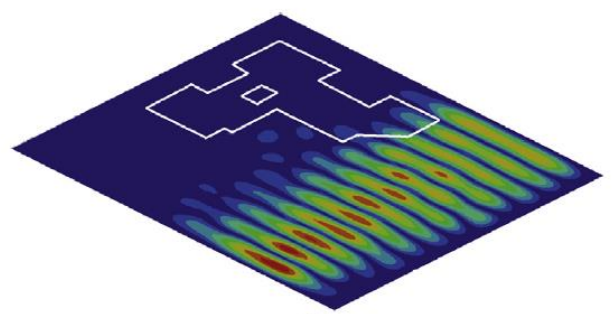

(a)

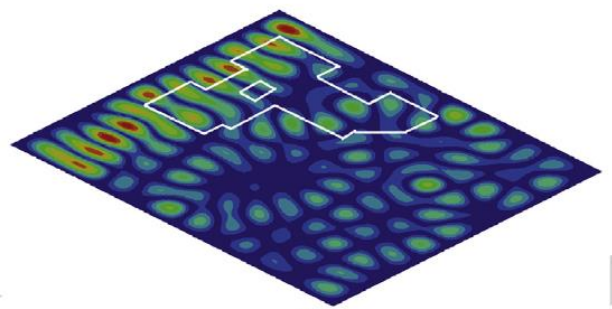

(b)

Figure 4. Example of 2 modeshapes of plate for the viscol configuration: (a) at $1584 \mathrm{~Hz}$; (b) at $1828 \mathrm{~Hz}$.

The influence of the dissipative material on the intermodal coupling loss factors and on the modal energy distribution for the cavity are illustrated in Fig. 5, by comparing these quantities between the bare and visco 2 configurations. These results concern the $1 \mathrm{kHz}$ third octave band for which the bare plate and the visco 2 plate have 19 and 18 modes, respectively, whereas the empty cavity has 21 modes. It can be seen that for the bare case, only two couples of plate-cavity modes ( i.e. couples $(4,3)$ and $(10,10)$ ) have significant intermodal coupling factor values. This shows that the coupling strength between these couples of modes is high compared to the other couples.

Although the number of subsystem modes is relatively high in this band, few of them participate significantly in the energy sharing between the two subsystems. This is in particular due to the fact that the frequency coincidences play a significant role when the damping is low. On the contrary, for the visco 2 configuration, a large number of couples have significant values for the intermodal coupling factors and participate in the energy sharing between the plate and the cavity. Almost all the cavity modes are significantly coupled to at least one

Page 4 of 8 plate mode. As observed in Fig. 4, the result is that the distribution of the modal energies of the cavity is more uniform for the visco 2 configuration than for the bare configuration: a variation of $35 \mathrm{~dB}$ for the bare configuration compared to $20 \mathrm{~dB}$ for the visco 2 configuration. Moreover, a considerable decrease of the highest values of the intermodal coupling factors for the visco 2 case compared to the bare case. It results more cavity modes are significantly coupled with the plate modes for the visco 2 case, but with less intensity.

Subsystem energies are finally obtained by summing the modal energies of each subsystem. For the $1 \mathrm{kHz}$ third octave, the energy level of the cavity is estimated to: $103 \mathrm{~dB}$ (ref. $10^{-12} \mathrm{~J}$ ) for the bare configuration, $92 \mathrm{~dB}$ for the viscol configuration and $89 \mathrm{~dB}$ for the visco 2 configuration.

The energy difference between the bare and the visco configurations may be directly related to the difference observed on Fig. 3 for the modal damping loss factors. The effect of one viscoelastic patch is very significant (i.e. cavity energy reduced of $10 \mathrm{~dB}$ ) whereas adding a supplementary one permits a supplementary decrease of cavity energy of around $3 \mathrm{~dB}$, only .
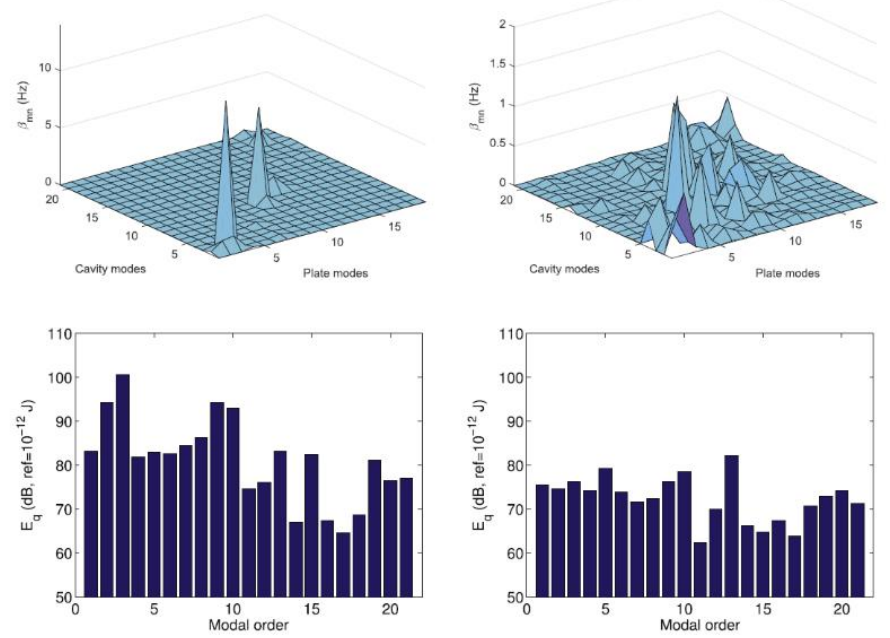

Figure 5. SmEdA results for the third-octave band $1 \mathrm{kHz}$ band: upper, modal coupling loss factors; lower, modal energy distribution of the receiving cavity. Two configurations: left, bare case ; right, visco2 case.

Results have been presented considering viscoelastic patches on the panel. The same type of results have been obtained considering a fibrous material inside the cavity. These results and a comparison with a laboratory experiment can be found in [7]. 


\section{III-Truck Cabin Modelling}

\section{III.1 Presentation of the industrial case}

In order to assess the accuracy of the SmEdA method for predicting the vibroacoustic behaviour of the concept lighten cabin of the CLIC project, an experimental validation study was carried out previously on an existing cabin supplied by the industrial partners (see figure (6)). One presents some results of this study in this section. The objective was to predict the pressure level inside the cabin (globally) and on the driver ears when a mechanical force excited a truck rail. The cabin was based on four air suspensions which allowed us to decouple it from the ground for frequencies above $20 \mathrm{~Hz}$. A hammer impact was used to excite the left rail on the back part, close to an air suspension as shown on figure 6 . An accelerometer was used for measuring the vibratory acceleration at a point close to the excitation point (on the rail) and different microphones were used for measuring the pressure at different points inside the cavity and at the driver ear position.
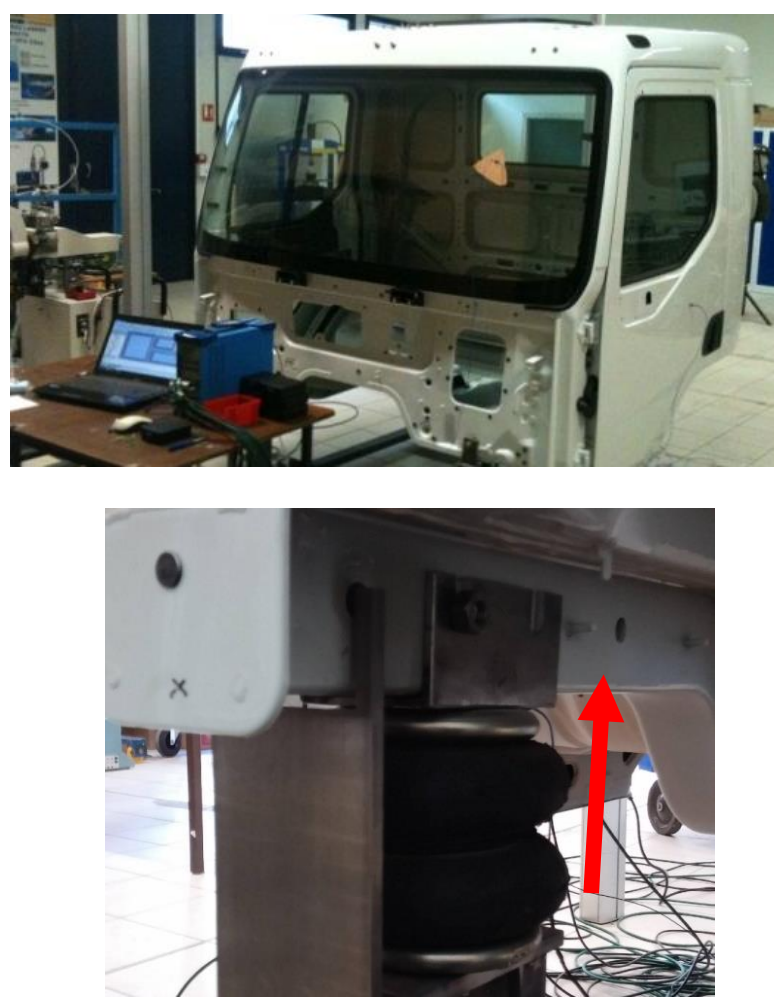

Figure 6. Upper, experimental set-up on the truck cabin; Lower, air suspension fixed the truck rail and force of excitation.

Two types of dissipative treatments had been considered (see figure 7): viscoelastic patches (called "IFF in French) stuck on the cabin floor and an acoustic panel composed of fibrous materials (felt) positioned on the rear of the cabin (see figure 7). The acoustic panel is composed of two parts, depending of the compression of the felt. Measurements inside the cabin were achieved with and without the acoustic panel.

Page 5 of 8

\section{III.2 SmEdA modelling}

For the numerical simulation, we supposed that the acoustic radiation into the cabin is dominated by the floor radiation. Then, we neglected the contributions of the walls and the roof in the modelling. These structures were considered as rigid and simply supported boundary conditions were imposed at the edges of the floor.

The viscoelastic patches are identical to the ones considered in section 2. The mechanical characteristics of the viscoelastic material were measured with the dynamic mechanical analyser and the equivalent single layer parameters of the multilayer were obtained using MOVISAND. For the fibrous materials, an acoustic tube with the two cavities method was used to estimate the equivalent characteristic impedance and the equivalent wavenumber of each felt. The equivalent fluid parameters (i.e. complex density and complex celerity) were deduced in function of frequency and averaged for each third octave band.
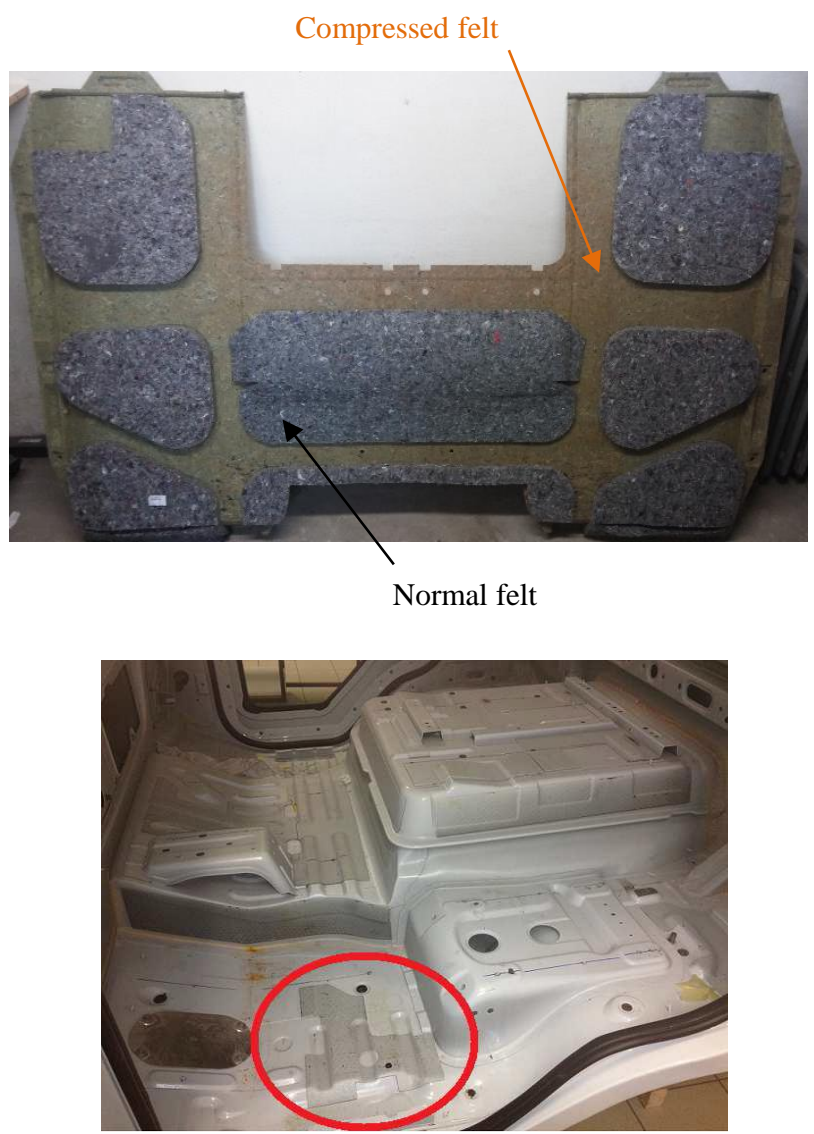

Viscoelastic pad

Figure 7. Upper, fibrous materials used to compose the rear panel; Lower, viscoelastic patches on the floor.

The acoustic cavity of the cabin with the porous materials and the floor structure with the viscoelastic patches were meshed by finite elements (see figure 8). The porous materials were represented by $3 \mathrm{D}$ TETRA elements. A fluid property taking the physical parameters estimated with the two cavities method into account was assigned to these elements. The viscoelastic patches were represented by $2 \mathrm{D}$ 
QUAD elements with a shell property taken the MOVISAND equivalent parameters into account. The FE models of the cavity and of the floor were composed of $3 \mathrm{Kdof}$ and $1 \mathrm{Mdof}$, respectively. The two meshes were not coincident at the coupling surface between the floor and the cavity. A projection method was used for the calculation of the intermodal works from the modeshapes of each subsystem [9]. As the parameters of the equivalent models (for the viscoelastic patches and the fibrous material) are frequency-dependant, finite element models were generated for each third octave band. The averaged values of the equivalent parameters were assigned to the finite elements related to the equivalent model. Modal analysis were performed using MSC/NASTRAN code. The process was automated as it was repeated for each third octave band of interest. For the different frequency bands, the MATLAB program allowed: (a), creating the input NASTRAN data file with the appropriate properties for the elements corresponding to the equivalent material; (b), running the NASTRAN calculation, and; (c), exporting the NASTRAN outputs in a MATLAB file. The data saved in the MATLAB file are the resonance frequencies, the mode shapes for the nodes belonging to the coupling surface and the modal damping loss factors. The SmEdA calculations were then performed under the MATLAB environment: first, the intermodal works were calculated from the modeshapes and the projection method (see illustration figure 9); second, the modal coupling loss factors were estimated using Eq. (2) with the intermodal works, the modal damping loss factors, and the modal frequencies; third, the injected power were calculated using Eq. (3); fourth, the modal energies were deduced from Eq. (1) with the quantities calculated previously; finally, the spatial mean square pressure inside the cavity was deduced from Eq. (4-5) and the pressure at the ear drivers was estimated using the process described in [10].
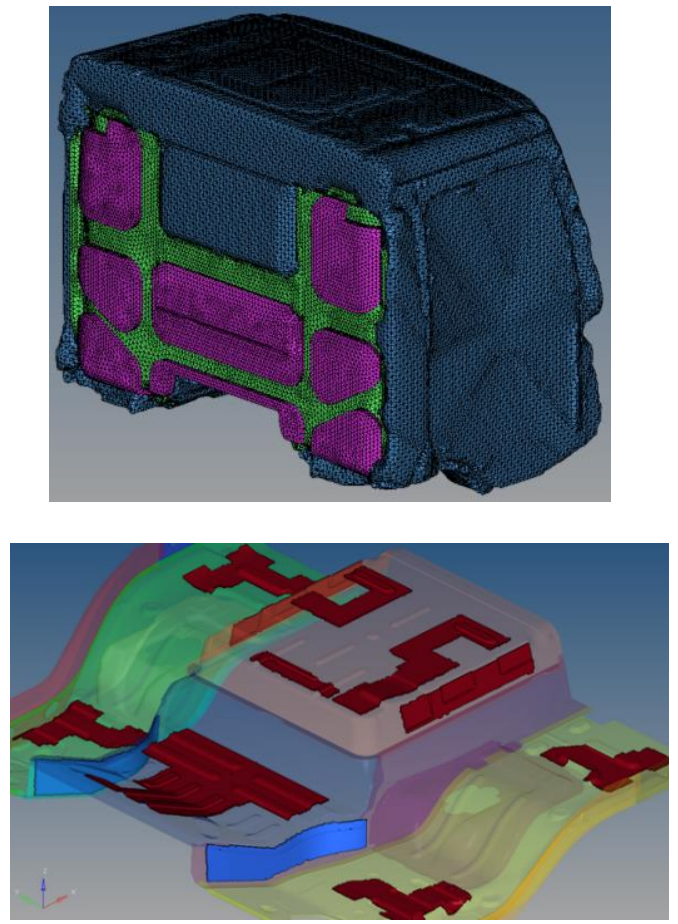

Figure 8. Upper, FEM of the cavity with the fibrous material. Green, compressed felt; pink, normal felt. Lower, FEM of the floor with the viscoelastic patches (indicated by strong red color).

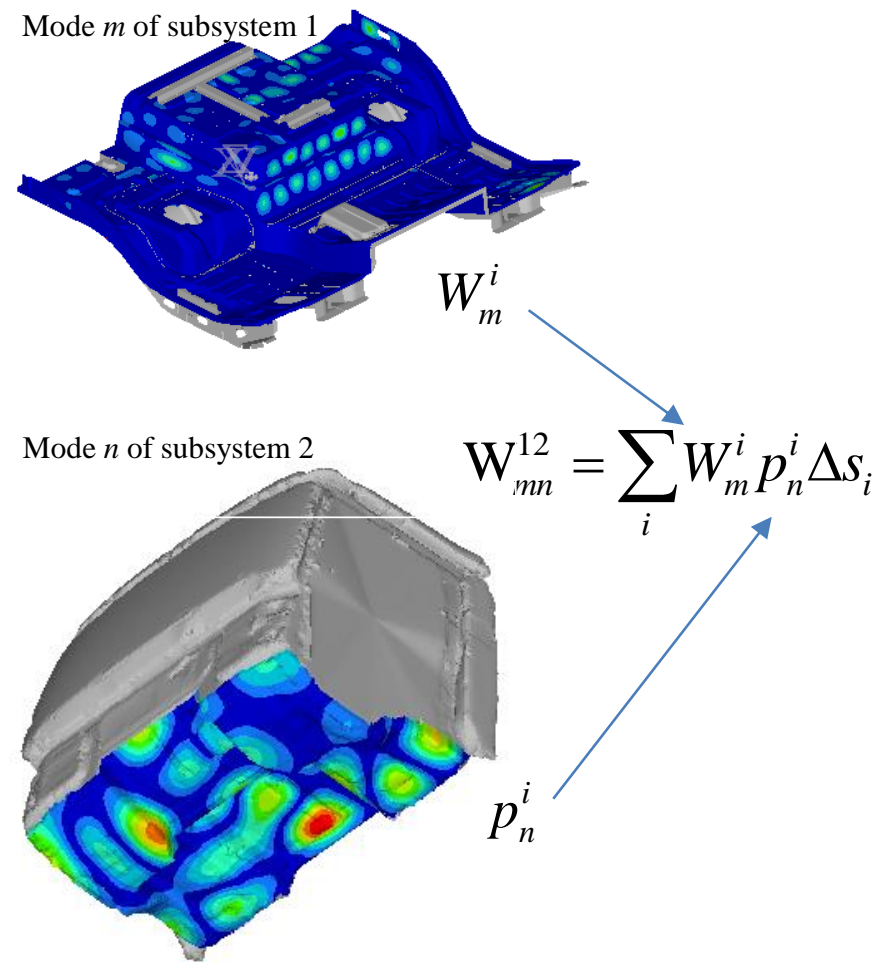

Figure 9. Illustration of the intermodal work calculation between a floor mode and a cavity mode.

\section{III.3 Results and comparison with the measurements}

Before analysing the SmEdA results, the acceleration level at the excitation point was calculated by FEM considering the floor model only. The result is compared to the measurement on Fig. 10. Globally, one observes a relatively fair agreement between the two results: the tendency is well respected and the discrepancies are lower than $2 \mathrm{~dB}$ in the lower part of the frequency band. However, one notes significant discrepancies (between 5 and 13) for 4 third octave bands. The reliability of the FEM can be questioned. In particular, the boundary conditions applied on the floor edge can be not appropriated. As the injected power by the force may be directly related to the acceleration at the force point, one can expect to observe discrepancies between the SmEdA and the experiment results for these frequency bands.

Figure 11 shows the pressure level inside the cabin calculated by SmEdA, with and without the damping patches. As it was not possible in practice to remove the patches, these results cannot be compared to experimental results. One notes however that the effect of the patches is very important (a decrease of around $10 \mathrm{~dB}$ on the pressure level inside the cavity). 


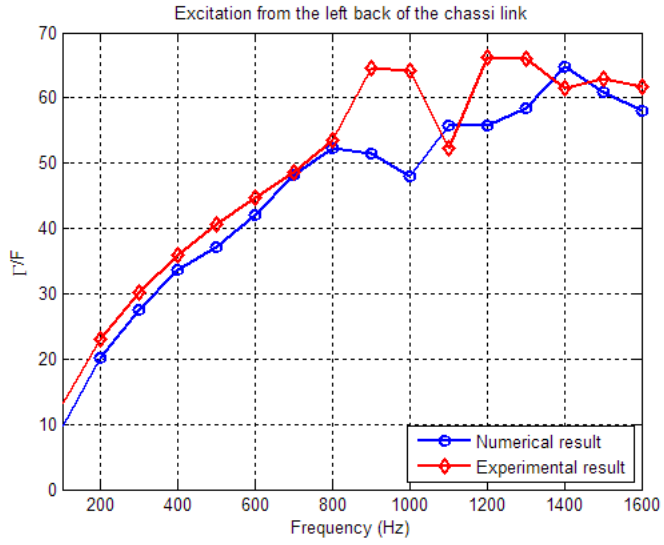

Figure 10. Acceleration level at the excitation point: comparison between the FEM result and the experimental measurements.

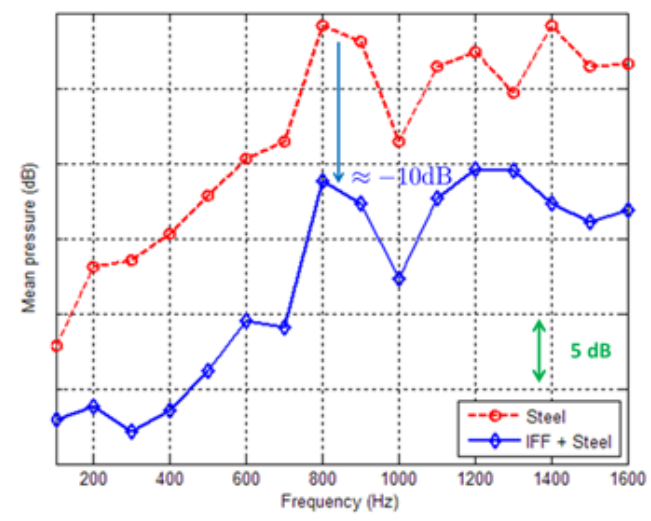

Figure 11. Global pressure level inside the cabin with and without the viscoelastic (IFF) patches. SmEdA results. Configuration without the acoustic panel.

Figure 12 present the pressure level at the driver ears. One observes a good accuracy of the SmEdA modelling in general. Some discrepancies can be observed in the band $[800 \mathrm{~Hz}-1200 \mathrm{~Hz}]$, that correspondd to the band for which discrepancies have been observed on the acceleration level (figure 10). The pressure difference between the two ear positions is smaller in the SmEdA modelling than observed experimentally. This can be expected as SmEdA is not adapted for describing small variations of the pressure/vibration field. The process described in [9] is well adapted when a subsystem exhibit a small number of modes (i.e. when the vibratory response is dominated by the response of few modes) or when a subsystem is non-homogeneous (i.e. with significant spatial variations of the vibratory field).

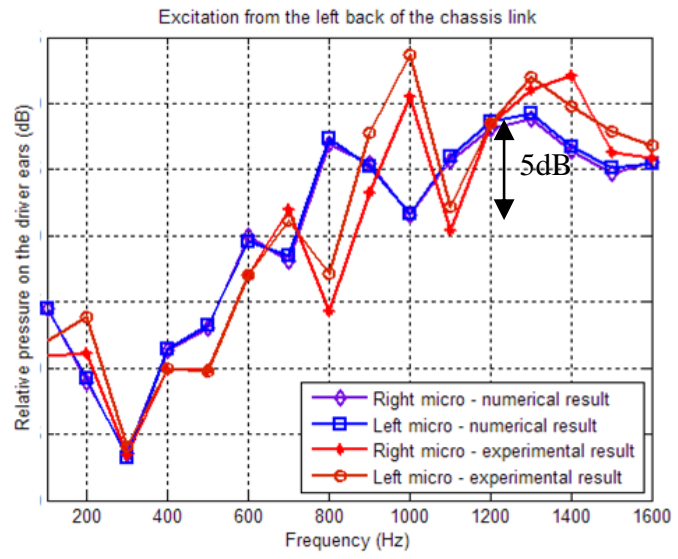

Figure 12. Pressure level on the driver ears: comparison between the SmEdA results and the measurements. Configuration without the acoustic panel.

Figure 13 shows the difference of the global pressure level between the cabin with and the cabin without the acoustic panel at the rear of the cabin. The global tendency is respected. Excepted for the third octave band $1 \mathrm{kHz}, \mathrm{SmEdA}$ predict correctly or slightly underestimate the effect of the panel. Even if these comparisons are not fully satisfactory, they show that the SmEdA model can take into account the effect of the dissipative material. A particular study should be carry out to define the most appropriate boundary conditions for the floor. Another way would consist to model the entire cabin. It would certainly lead to better results because the issue raised by the boundary conditions of the floor would be relaxed.

Page 7 of 8 

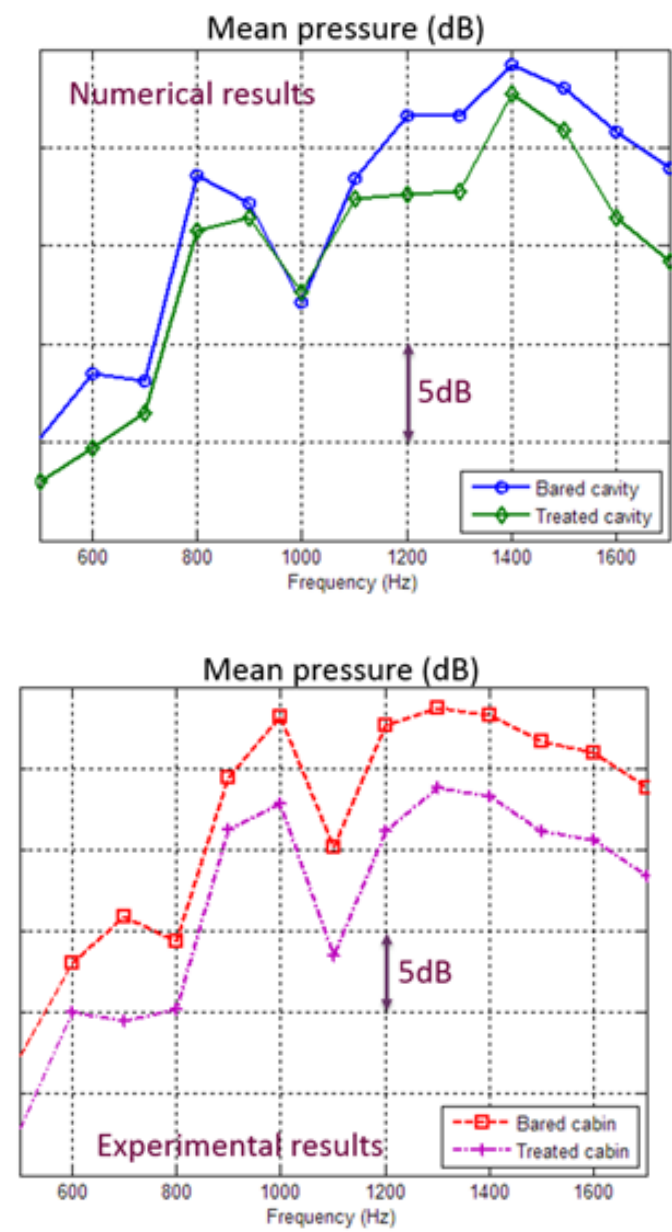

Figure 13. Global pressure level inside the cabin: upper, SmEdA results; lower, measurements.

\section{IV-Conclusions}

An approach based on the SmEdA method has been proposed to predict the vibroacoustic behavior in the mid frequency range of a truck cabin including the dissipative materials (i.e. visco-elastic layers, acoustic absorbing materials). The calculation process requires to achieve modal analysis from Finite Element models of the uncoupled-subsystems including the dissipative treatments. These latter are represented with simplified equivalent models. This has the advantages to easier their introduction in the FE models in the practical applications, to reduce the computing times compared to a full model and to be well adapted for re-analyzing in an optimization purpose. A key point of this approach relies on the use of the Galerkin procedure for estimating the modal damping loss factors of the treated subsystems. A NASTRAN DMAP program allowed estimating these factors in the same run as the modal analysis. The application on the truck cabin has shown the feasibility of the approach for predicted the sound pressure up to $2 \mathrm{kHz}$. Comparisons with experiment results were acceptable even if additional investigations are necessary to completely assess the ability of the approach for predicting the vibroacoustic behaviour of an industrial structure like the truck cabin.

Page 8 of 8

\section{References}

1. R.H. Lyon, Statistical Energy Analysis of Dynamical Systems: Theory and Application, MIT press Cambridge, (1975)

2. A Le Bot and V. Cotoni. Validity diagrams of statistical energy analysis. Journal of Sound and Vibration 329 (2) (2010) 221235.

3. L. Maxit, J.L. Guyader, Estimation of SEA coupling loss factors using a dual formulation and FEM modal information, part I: theory, Journal of Sound and Vibration, 239 (2001) 907-930.

4. L. Maxit, J.L. Guyader, Extension of SEA model to subsystems with non-uniform modal energy distribution, Journal of Sound and Vibration, 265 (2003) 337-358.

5. L. Maxit, Statistical modal Energy distribution Analysis (SmEdA), UP2HF summer school, Lyon France, 2016 https://up2hf.sciencesconf.org/conference/up2hf/III_Up2HF_La urent_Maxit.pdf

6. L. Maxit, K. Ege, N. Totaro, J.L. Guyader, Non resonant transmission modelling with Statistical modal Energy distribution Analysis, Journal of Sound and Vibration, 333 (2014) 499-519.

7. H.D. Hwang, L. Maxit, K. Ege, Y. Gerges, J.L. Guyader, SmEdA vibro-acoustic modelling in the mid-frequency range including the effect of dissipative treatments , Journal of Sound and Vibration, 393 (2017) 187-215.

8. J.L. Guyader, C. Cacciolati, "Viscoelastic properties of single layer plate material equivalent to multi-layer composites plate", INTER-NOISE and NOISE-CON Congress and Conference Proceedings 2007

9. Y. Gerges, H.D. Hwang, K. Ege, L. Maxit, C. Sandier, Vibroacoustic modelling of a trimmed truck cab in the mid frequency range. Proceedings of Internoise 2015, San Francisco, USA, August 2015.

10. N. Totaro, J.L. Guyader, Extension of the statistical modal energy distribution analysis for estimating energy density in coupled subsystems, Journal of Sound and Vibration, 331 (2012) 264-289.

\section{Contact Information}

L. Maxit (laurent.maxit@insa-lyon.fr)

J.L. Guyader (jean-louis.guyader@insa-lyon.fr)

K. Ege (kerem.ege@insa-lyon.fr)

Univ Lyon, INSA Lyon

Laboratoire Vibrations Acoustique

25 bis avenue Jean Capelle

69621 Villeurbanne cedex - France

\section{Acknowledgements}

This work was co-funded by the French government (FUI 12 Fonds Unique Interministériel) and European Union (FEDER - Fonds européen de développement régional). It was carried out in the framework of the LabEx CeLyA ("Centre Lyonnais d'Acoustique", ANR-10-LABX-60) and the research project CLIC ("City Lightweight Innovative Cab") labelled by LUTB cluster (Lyon Urban Truck and Bus), in partnership with Renault Trucks, Arcelor-Mittal, ACOEM, ALTRAN, FEMTO-ST (Univ. de Franche-Comté) and LVA (INSA de Lyon). 